\title{
Current Clinical Evidence for Korean Red Ginseng in Management of Diabetes and Vascular Disease: A Toronto's Ginseng Clinical Testing Program
}

\author{
Vladimir Vuksan ${ }^{1,2^{*}}$, John Sievenpipper ${ }^{1,2}$, Elena Jovanovski ${ }^{1,2}$, and Alexandra L. Jenkins ${ }^{1,2}$ \\ ${ }^{1}$ Clinical Nutrition Risk Factor Modification Center, St. Michael's Hospital, Toronto, ON, Canada \\ ${ }^{2}$ Departments of Nutritional Sciences and Medicine, Faculty of Medicine, University of Toronto, Toronto, ON, Canada
}

While ginseng is reported to have a wide array of applications, there is growing evidence for its indications in diabetes and vascular disease. A clear connection, however, has not been established between ginseng's composition, dose and its targeted efficacy in humans. We therefore developed and initiated the Korean Red Ginseng Clinical Testing Program for diabetes and vascular function which is an efficacy and safety-based clinical screening model for ginseng. The most efficacious sources, ginsenoside profiles, doses, and modes of administration were examined in sequential, acute, followed by long term, randomized-controlled trials to investigate the efficacy and safety profiles. This review discusses the current state of the clinical research of Korean red ginseng program conducted in Toronto, paving the way for the use of clinically selected ginseng and its ginsenoside fractions in the management of diabetes and vascular diseases.

Keywords: Korean red ginseng, Ginsenoside extract, Randomized controlled trial, Glycemic control, Vascular disease

\section{INTRODUCTION}

Cardiovascular disease (CVD) is the leading cause of mortality in industrialized nations. Likewise, diabetes, a major risk factor in CVD, is escalating to pandemic proportions. From 1995 to 2025 the prevalence of adult diabetes was predicted to increase by $27 \%$ in developed countries and $48 \%$ in developing countries [1].

Despite numerous preventative strategies and the growing armamentarium of medications available, the prevalence of impaired glucose tolerance, diabetes and its associated vascular complications continue to grow. Although oral anti-hyperglycemic agents have been shown to reduce microvascular complications, they have failed to deliver the anticipated macrovascular benefits in people with diabetes [2-6]. A meta-analysis showed that the insulin senitizer, rosiglitazone, although effective at improving glycemic control, as assed by $\mathrm{HbAlc}$, increased the risk of cardiovascular mortality by $40 \%$ in type 2 diabetes, a group already predisposed to increased premature cardiovascular death [5]. Three landmark clinical trials also recently demonstrated that intensive glycemic control by oral agents or insulin was of no benefit $[3,6]$ or even increased all cause mortality by $22 \%$ and cardiovascular mortality by $35 \%$ in people with poorly controlled type 2 diabetes [2]. These data indicate a clear and present need for more effective treatment strategies.

Evidence is emerging to support ginseng as a new approach to treat diabetes and its vascular complications. Prior to 2000, there was only a small group of flawed and poorly reported studies in humans to support the antidiabetic efficacy of ginseng [7]. The limited data prompted us to conduct a series of human trials to address systematically whether the glycemic lowering

(c) This is an Open Access article distributed under the terms of the Creative Commons Attribution Non-Commercial License (http://creativecommons.org/licenses/by-nc/3.0/) which permits unrestricted non-commercial use, distribution, and reproduction in any medium, provided the original work is properly cited.

Received 30 Nov. 2010, Revised 14 Dec. 2010, Accepted 16 Dec. 2010

*Corresponding author

E-mail: v.vuksan@utoronto.ca

Tel: +1-416-864-5525, Fax: +1-416-864-5538 
effects of ginseng were reproducible across different protocols and ginseng sources and whether ginsenosides, for which the most compelling evidence existed for biological activity, were mediating the glycemic effects. We also set out to investigate the discrepancy in available literature on ginseng effects on vascular parameters. We showed that ginseng had poor reproducibility across different source parameters and that ginsenosides were only weak predictors [8-11]. In the absence of a clear connection between its composition and efficacy, clinical screening was the only way to assure reliable benefit. This led us to develop an efficacy-based clinical screening model, in which the most efficacious ginseng sources, doses, and modes of administration were selected from sequential, "phase I", acute, single-bolus, randomized controlled trials and then applied in "phase II", longterm, randomized controlled trials to demonstrate the sustainability of efficacy and safety $[12,13]$. An example of this systemic evaluation concept was demonstrated for American ginseng use in diabetes [12-20].

The Korean Red Ginseng Clinical Testing Program has both a diabetes arm and a vascular function arm. This review discusses the current state of the clinical research in each arm of this Toronto based program.

\section{KOREAN RED GINSENG CLINCIAL DIABETES PROGRAM}

The clinical screening model is used to test whether the batch, preparation, dosing, and timing of a Korean red ginseng (KRG) source could be selected to have long-term efficacy using the same acute postprandial testing program [13]. This species was considered to be a good candidate for efficacy screening, as it is the only other type that has been reported to decrease glycemia in humans [21]. It also shares a similar protopanaxadol (PPD):protopanaxatriol (PPT) ginsenoside ratio with the selected batch of American ginseng used in our American Ginseng Clinical Testing Program $[8,12]$. We hypothesized that a batch of KRG selected will lower postprandial glycemia. Fractionation and extraction were applied to a single batch of KRG (Korea Ginseng and Tobacco Research Institute, Daejeon, Korea) to produce different preparations with a wide range in ginsenoside profiles. These included 3 preparations: KRG-rootlets, - root body, and -whole root $\mathrm{H}_{2} \mathrm{O}$ extract. We used this starting material to initiate a KRG testing program consisting of an acute and a longterm phase. A depiction is provided in Fig. 1. Sequential, "phase I", acute, single bolus, double-blind, randomized, placebo-controlled, multiple crossover, clinical trials were conducted, to identify an efficacious KRG preparation, dose, mode, and timing of administration. The dose range was from 2 to $6 \mathrm{~g}$, typically recommended by traditional Chinese medicine and practitioners, the mode of administration was a periprandial oral agent and timing was -40 -min prior to a meal, based on our prior clinical experience. The studies consisted of a preparation-finding trial (KRG-rootlets, -root body, and -whole root $\mathrm{H}_{2} \mathrm{O}$ extract), followed by a dose-finding trial ( $0[$ placebo], 2,4 , and $6 \mathrm{~g}$ ) of the most efficacious fraction. A $50 \mathrm{~g}$-oral glucose tolerance test (OGTT) protocol was used in which single boluses of the selected KRG preparations and doses were given as an oral agent -40 min preprandially to normoglycemic subjects. The preparation and treatment protocol gained from these acute studies was then applied to the safety and efficacy of KRG intervention (SAEKI) trial, a longterm, double-blind, "phase II" randomized controlled trial, conducted by our group in subjects with type 2 diabetes.

\section{Sequential acute randomized controlled trials Acute trial 1}

The first, acute, preparation-finding, "phase I", randomized controlled trial [22] assessed the efficacy of single $6 \mathrm{~g}$ doses of the Korean red ginseng-rootlets, root body, and -whole root $\mathrm{H}_{2} \mathrm{O}$-extract given as an oral agent -40 min preprandially in lowering the glycemic response to a 50 g-OGTT relative to placebo in 7 normoglycemic subjects (Fig. 1, panel 1). A wide variation in the ginsenoside profiles was achieved across the 3 root fractions. This variation coincided with differential effects, although the effects did not appear to be related to differences in the PPD:PPT ginsenoside ratio. Korean red ginseng-rootlets decreased the glycemic response to the $50 \mathrm{~g}$-OGTT at 90-min compared with placebo, while neither the KRG-root body nor -whole root $\mathrm{H}_{2} \mathrm{O}$ extract affected glycemia significantly. This was reflected in a significant $29 \%$ reduction in area under the curve (AUC) by the KRG-rootlets compared with placebo. We concluded that KRG rootlets, as the most efficacious preparation, would advance to the next step in the acute phase of the clinical testing program.

\section{Acute trial 2}

In the second acute, dose-finding, "phase I", randomized controlled trial [22], 12 normoglycemic subjects received single doses of $0 \mathrm{~g}$ (placebo), $2 \mathrm{~g}, 4 \mathrm{~g}$, and $6 \mathrm{~g}$ of the selected KRG-rootlets following the same $50 \mathrm{~g}$ OGTT protocol described for the preparation-finding tri- 


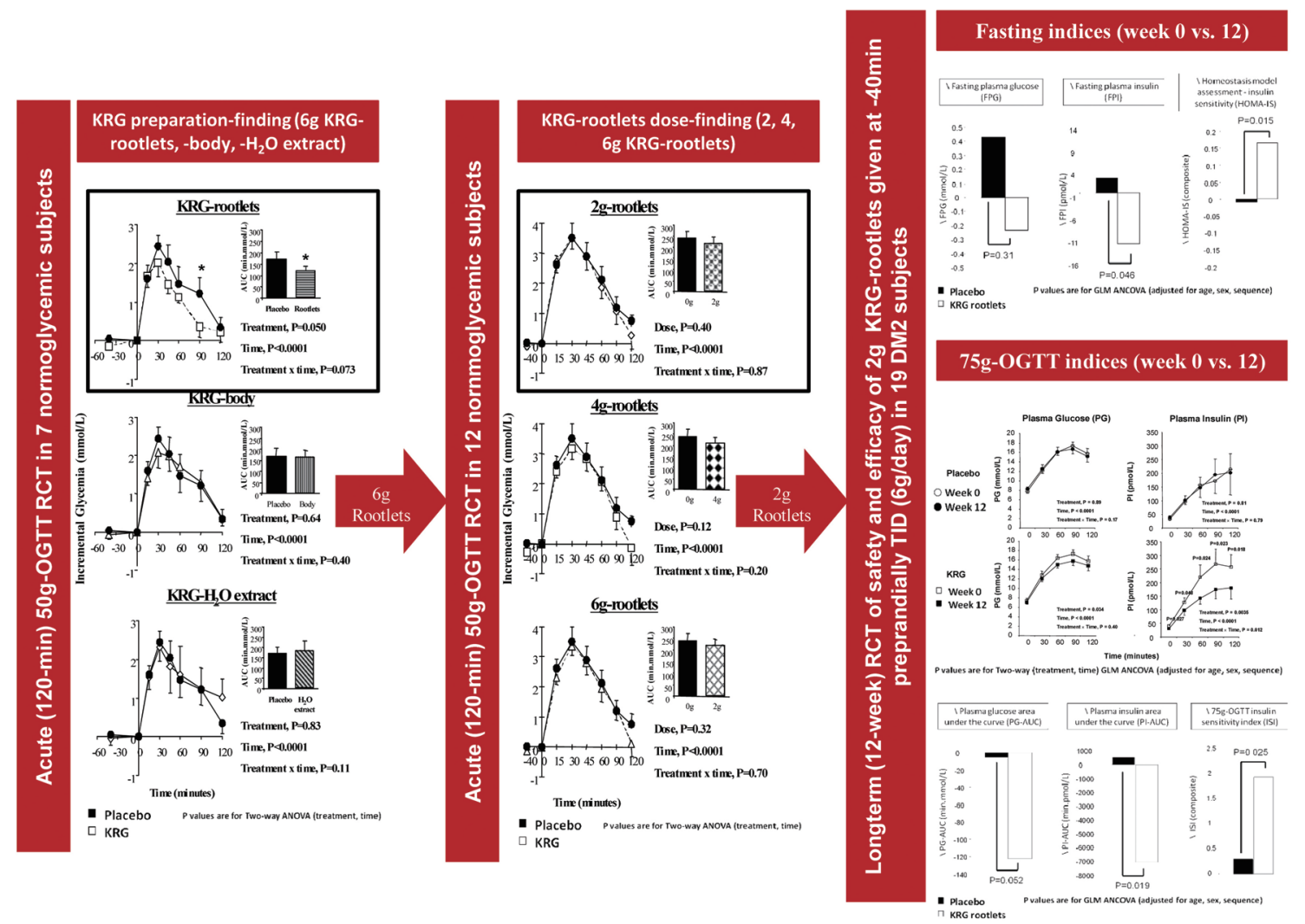

Fig. 1. Schematic of the Korean Red Ginseng Clinical Testing Program for diabetes. The program consisted of a series of acute, "phase I", single-bolus, randomized, controlled, crossover trials to evaluate the optimal preparation and dose derived from a single batch of Korean red ginseng (KRG) in normoglycemic subjects, followed by a longterm, "phase 2" randomized controlled trial, in which this information was applied in subjects with type 2 diabetes (DM2). Acute effects were assessed on the glycemic response to50g-oral glucose tolerance test (OGTT) compared with placebo. Each study informed the next regarding the most efficacious preparation and dose to advance (written in arrows). Although there was no effect of any of the 3 doses individually versus placebo, the mean of the 3 doses reduced the glycemic response to the 50g-OGTT significantly compared with placebo, implying that the 3 doses were equally efficacious with the lowest dose advanced. Asterisk indicates significant differences $(p<0.05)$. A $p$-value is for independent and interactive effects assessed by 2-way ANOVA. RCT -Randomized Controlled Trial; TID, administered three times/day. Sievenpiper et al. [33] and Vuksan et al. [23]

al (Fig. 1, panel 2). A significant effect of KRG rootlets treatment (mean of 3 doses) was found. The mean of all 3 doses decreased mean postprandial incremental glycemia by $17 \%$ compared with placebo. This was reflected in the AUC, in which there was a tendency for the mean of all 3 doses of the KRG-rootlets to decrease the AUC by $14 \%$ compared with placebo. There was, however, no effect of individual doses compared with placebo. It was concluded that the selected KRG-rootlets decreased the glycemic response to the $50 \mathrm{~g}$-OGTT irrespective of dose. The selected KRG-rootlets were equally efficacious in lowering postprandial glycemia at doses from $2 \mathrm{~g}$ to $6 \mathrm{~g}$ when given as an oral agent $-40 \mathrm{~min}$ preprandially.

Overall conclusions were drawn from the acute phase to guide the longterm phase of the clinical testing program. The two "phase I", acute, randomized controlled trials were successful in identifying the most efficacious preparation (KRG rootlets), dose ( $\mathrm{g}$ ), and mode of ad- ministration (oral agent given at -40 min preprandially) for achieving reproducible reductions in acute postprandial glycemia. Application of this treatment protocol was indicated for the "phase II" randomized controlled SAEKI trial.

\section{Long term randomized controlled trial (SAEKI trial)}

The treatment protocol identified by the sequential acute preparation- and dose-finding studies was applied to the longterm, "phase II", double-blind, randomized, placebo-controlled, crossover SAEKI trial (Fig. 1, panel 3) [23]. Nineteen type 2 diabetic subjects received $2 \mathrm{~g}$ placebo or the selected KRG rootlets as an oral agent -40 min prepranldially three times/ day (6 g/day) for 12 weeks, while maintained on their conventional diabetes treatment. Fasting plasma insulin and 75 g-OGTT derived AUC plasma insulin were significantly decreased on the selected KRG rootlets treatment compared with 
placebo. This occurred while fasting plasma glucose was unchanged and $75 \mathrm{~g}$-OGTT derived AUC plasma glucose was significantly decreased. The combination was reflected in an identical 33\% increase in both the homeostasis model assessment (HOMA) and the $75 \mathrm{~g}$ OGTT derived insulin sensitivity indices (75 g-OGTT insulin sensitivity index) on the selected KRG treatment compared with placebo. These benefits occurred without increasing adverse events or altering hepatic, renal, haemostatic, or blood pressure function. We concluded that our KRG clinical testing program successfully identified a KRG preparation (rootlets), dose (2 g), and mode of administration (oral agent given -40 min preprandially) that resulted in sustainable improvements in longterm glucose and insulin regulation safely beyond conventional treatment in type 2 diabetes.

\section{KOREAN RED GINSENG CLINICAL VASCULAR PROGRAM}

There is convincing pre-clinical evidence that KRG may benefit vascular function. Fractions of KRG have shown hemodynamic effects in animal and aortic ring studies [24-32], including vasodilatation, platelet inhibition and nitric oxide release stimulation by ginsenosides, especially $\operatorname{Rg}_{3}[25,28,29,31]$, and enhancement of production of nitric oxide in vitro by polysaccharides [33,34]. Corresponding clinical evaluations of KRG and its components, however, are limited. Clinical evidence on the hemodynamic properties of ginseng has demonstrated either neutral or moderate blood pressure (BP) lowering effects [32,35-37]. Even in the SAEKI trail our selected KRG rootlets showed a neutral effect on blood pressure as assessed by office BP and 24h-ambulatory BP measurements in people with type 2 diabetes [23]. Therefore there is much discrepancy in the literature on blood pressure effects of Korean ginseng despite stronger preclinical evidence on its vasodilatory effects. A vascular benefit, however, can occur without a detectable effect on blood pressure. Vasodilating agents have generally been implicated as having little direct effect on elastic arteries and reduction in initial stroke pressure (systolic pressure) but can markedly lower ventricular after load by decreasing muscular artery stiffness and wave reflection amplitude [38,39]. These beneficial effects on arterial waves can occur with or without a reduction in conventional office blood pressure [39]. To determine whether KRG has clinical vasoactive effects beyond brachial $\mathrm{BP}$, augmentation index (AI), a non invasive measure of arterial wave reflection constructed from pulse wave analysis, flow mediated dilatation (FMD) a measure of blood flow, and central BP, all independent markers of increased total and cardiovascular mortality $[40,41]$, would provide additional clinical and prognostic information concerning hemodynamic effects. We therefore undertook the KRG clinical testing program for vascular function assessed both by conventional BP measurements and by FMD, AI and central BP. We systematically investigated effects of KRG from an efficacy based as well as component based perspective.

\section{Sequential acute randomized controlled trials: blood pressure studies}

The KRG clinical testing program for vascular function mirrored the corresponding program for diabetes.

\section{Acute trial 1}

In the first vascular function trial, two of the same preparations from the same batch of KRG (rootlets and body) were studied using a "phase I", acute, single-bolus, randomized, crossover, double-blind, placebo-controlled, clinical, BP protocol [42]. The rootlets and body of the same ginseng batch were selected due to a difference in the total ginsenoside content between the rootlets and the root ( $84 \%$ higher in rootlets) while minimizing the environmental and genetic differences between test samples. Seventeen nondiabetic, non hypertensive individuals arrived at our clinic after a 10 to 12 -h fast on 3 different mornings without taking their antihypertensive drugs that morning. Individuals first had office BP measures taken until three consecutive systolic and diastolic readings differed by $<5 \mathrm{mmHg}$. Subjects were then fitted with a SpaceLabs ambulatory BP monitor (ABPM), which measured BP every 5-min for the first $30 \mathrm{~min}$ - the average of these 7 measures constituted the baseline BP reading. Individuals then consumed $3 \mathrm{~g}$ of encapsulated cornstarch (placebo) or KRG-body or rootlets in random order on separate days. BP was recorded with the ABPM every 10 -min for $160 \mathrm{~min}$. At 60 -min, a standardized breakfast was consumed to similate typical physiological conditions. The change in systolic and diastolic BP at each time-point relative to baseline was determined for each individual and averaged. We found that $\mathrm{KRG}$ rootlets significantly reduced systolic BP at 120-min compared with placebo. KRG body, containing less total ginsenosides, showed no effect on BP. We concluded that, similar to the data from the diabetes program, the data from the vascular function program demonstrated that the KRG rootlets were the more efficacious root fraction, by effectively lowering BP in 
healthy individuals. Ginsenosides were implicated in these effects.

\section{Acute trial 2}

In order to explore further the role of ginsenosides in BP effects, the second vascular function trial [43] was designed. The ginsenoside $\mathrm{Rg}_{3}$ was selected for study, as it had been directly implicated as the most potent vasodilator among ginsenosides supported by the most robust evidence $[25,28,29,31]$. Using a "phase I", acute, single bolus, randomized, placebo-controlled, 4-period crossover clinical trial design, the effect of KRG extracts with escalating levels of ginsenoside $\mathrm{Rg}_{3}$ on BP. After a 12-h fast on 4 separate days, 10 drug-treated subjects with hypertension and 3 drug-naive subjects with highnormal BP randomly consumed $500 \mathrm{mg}$ of placebo $(0$ $\left.\mathrm{mg} \mathrm{Rg}_{3}\right)$ or KRG extract with low $(0.27 \mathrm{mg})$, medium $(0.81 \mathrm{mg})$, or high $(2.43 \mathrm{mg})$ doses of ginsenoside $\mathrm{Rg}_{3}$ in identical capsules at time 0-min. Drug-treated individuals refrained from taking antihypertensive agents on test days. At 60-min, a 356-mL (380-calorie) Ensure breakfast was consumed. BP was measured with ABPM (Accutracker II; Suntech Medical Instruments, Raleigh, $\mathrm{NC}$, USA) every $5 \mathrm{~min}$ for the first $20 \mathrm{~min}$ before time 0 -min (the mean of this represented baseline BP) and every 15 minutes for $180 \mathrm{~min}$ after the consumption of placebo or KRG extract. We found that the KRG extract with the medium $\mathrm{Rg}_{3}$ dose resulted in large systolic and diastolic BP reductions relative to baseline at 135-min. Consumption of the low and high dose $\mathrm{Rg}_{3}$ extracts showed no significant effect on BP. It was concluded that escalating the dose of ginsenoside $\mathrm{Rg}$ up to $0.84 \mathrm{mg}$ in KRG extracts can result in profound acute blood pressure effects.

\section{Acute trial 3: flow mediated dilatation study}

While mild or neutral blood pressure lowering effects were observed in the first trial, the subsequent objective was to evaluate the effect of ginseng treatment on more sensitive vascular markers to provide additional information on potential waveform modulation beyond systolic and diastolic blood pressure parameters. Our next step therefore was to explore further the effects of the KRG rootlets identified in the first acute blood pressure trial on these more sensitive markers of vascular function, starting with FMD. Our further objective was also to investigate the direct contribution of its extracted ginsenoside and polysaccharide fractions [44]. The ginsenoside and polysaccharide fractions were extracted at a dose bioequivalent to that provided by $3 \mathrm{~g}$ of the KRG rootlets. This approach allowed evaluation of the direct influence of major ginseng fractions on FMD, as well as the comparison of their effects with those of the unprocessed rootlets from which they had been fractionated. FMD studies of the effects of the selected KRG rootlets and its ginsenoside and polysaccharide fractions were undertaken. Using a "phase I", acute, single-bolus, randomized, crossover design, 17 healthy subjects received the selected KRG rootlets $(3 \mathrm{~g})$, ginsenoside extract $(0.21$ $\mathrm{g}$ ), polysaccharide extract $(0.22 \mathrm{~g})$, or placebo (cornstarch) in random sequence. Extracted polysaccharides and ginsenosides were delivered at doses identical to those found in $3 \mathrm{~g}$ of root. Flow mediated dilatation at the brachial artery was assessed at baseline and at 90and 180-min post treatment. We found that the selected KRG significantly improved FMD at 180 min compared with placebo. The ginsenoside extract produced a comparable shear stress-induced vasodilation compared to control, whereas the polysaccharide fraction did not (Fig. 2A). The ginsenoside extract and whole rootlets did not significantly differ in the changes induced on the vascular endothelium. It was concluded that the selected KRG root acutely improved vascular endothelial function in healthy individuals compared to control, an effect partly attributed to its ginsenoside containing fraction which was high in ginsenoside $\mathrm{Rg}_{3}$.

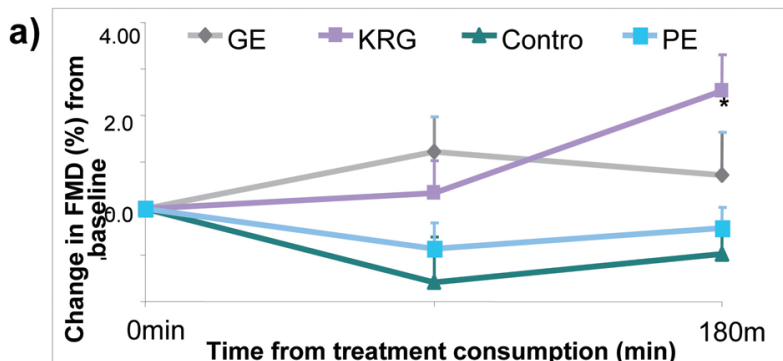

b)

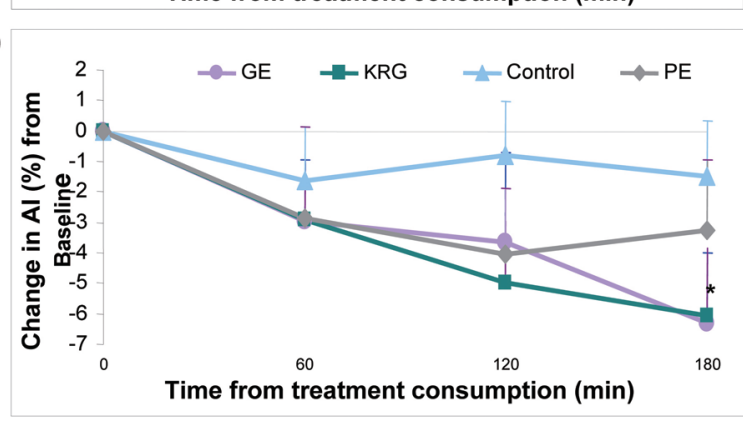

Fig. 2. Effect of Korean red ginseng (KRG), ginsenoside extract (GE) and polysaccharide extract (PE) versus Control on a) flow mediated dilatation (FMD) and b) radial augmentation index ( $\mathrm{Al})$, a measure of arterial stifness. Each point represents the post treatment change in a) FMD and b) $\mathrm{Al}$, respectively, relative to baseline. Data are mean \pm SEM. ${ }^{*} p<0.05$. 
Acute randomized controlled trial 4: augmentation index study

In the same population of 17 healthy subjects, our subsequent objective was to further investigate vasoactive efficacy of the selected KRG rootlets on pulse wave reflection [44], and compare the effects of rootlets to its two major isolated fractions: ginsenosides and polysaccharides. Pulse wave reflection is a marker of arterial stiffness and provides additional information on the workload of the heart, coronary blood flow, and mechanical integrity of arteries. Radial AI, which characterizes pulse wave reflection, was measured by applanation tonometry (HEM-9000AI; Omron Healthcare Co., Kyoto, Japan) at baseline and at $1 \mathrm{~h}, 2 \mathrm{~h}$, and $3 \mathrm{~h}$ post treatment. The ginsenoside fraction significantly lowered radial AI indicating a decrease in pulse wave reflection, whereas the polysaccharide fraction again did not (Fig. 2B). We concluded that the selected KRG rootlets and their ginsenoside fraction significantly improved arterial stiffness, implicating the ginsenosides (noted to be high in ginsenoside $\mathrm{Rg}_{3}$ ) as the principal pharmacologically active components of the root. Taken together with the FMD study, these data support further investigation into KRG's microcomponent fractions, especially its ginsenoside fraction and provides a better basis for ginseng standardization for vascular function related to improved endothelial function and arterial stiffness.

\section{Acute randomized controlled trial 5: $\mathrm{Rg} 3$ ginseng vascu- lar effects}

Subsequent to observed favorable vasoactive effects of KRG rootlets and its ginsenoside fraction, our further aim in the stepwise assessment was to concentrate and further isolate the ginsenoside that may possess vasodilating properties. As ginsenoside $\mathrm{Rg}_{3}$ was supported by pre-clinical evidence to be the most effective vasoactive component of ginseng and was previously found to have a mild effect on brachial blood pressure, we set to in-

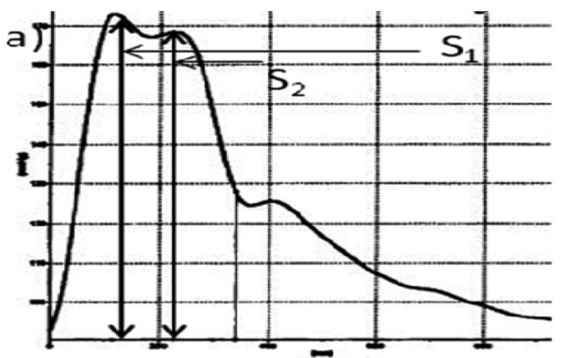

vestigate vascular effects of a $10 \% \mathrm{Rg}_{3}$-enriched $\mathrm{KRG}$ fraction. The sample used in this trial was manufactured by the BTGin Co. (Okcheon, Korea), using chemo-enzymatic process (in situ) and it has a significantly higher concentration of ginsenoside $\operatorname{Rg}_{3}$ (20 times higher) compared to the sample previously used in our program. Our specific objective therefore, was to evaluate the acute effects of $10 \% \mathrm{Rg}_{3}$-enriched $\mathrm{KRG}$ on arterial stiffness, peripheral and central systolic BP (SBP) and diastolic BP (DBP) in healthy adult volunteers.

Using a double-blind, randomized, crossover design, 23 individuals (SBP, $113 \pm 3 \mathrm{mmHg}$; DBP, $70 \pm 2 \mathrm{mmHg}$ ) were administered $400 \mathrm{mg} 10 \% \mathrm{Rg}_{3}$-enriched KRG extract or $400 \mathrm{mg}$ corn starch placebo. Aortic augmentation index (AIx) and central BP were measured using applanation tonometry by radial pulse wave analysis and peripheral BP was evaluated oscillometrically. Measurements were taken at baseline and 1,2 and 3hrs posttreatment. Compared to placebo, there was a significant reduction in peripheral mean arterial pressure, peripheral DBP, and central SBP and DBP 3 hrs post-treatment (Fig. 3). Central DBP and central mean arterial pressure were significantly lower at $1 \mathrm{~h}(p<0.05)$ and $3 \mathrm{~h}(p<0.05)$ posttreatment. Compared to placebo, AIx was significantly decreased post-treatment, with over $9 \%$ absolute reduction at $3 \mathrm{hrs}$ time point (Fig. 4). This study is the first to investigate the efficacy of $\mathrm{Rg}_{3}$-enriched $\mathrm{KRG}$ extract and its effects on arterial stiffness and central and peripheral blood pressure. This study provide preliminary evidence of acute efficacy of an $\mathrm{Rg}_{3} \mathrm{KRG}$ extract on indeces of peripheral and central vascular function in healthy, normotensive, young adults. Further clinical testing is still required as this extract may be more potent in higher-risk subjects.

As with the KRG testing program for diabetes, confirmation of these findings and their sustainability needs to be established by "phase II", long term randomized clinical trials. This exciting new prospect of applying $\mathrm{Rg}_{3}-$ enriched ginseng in the long -term clinical trial, together

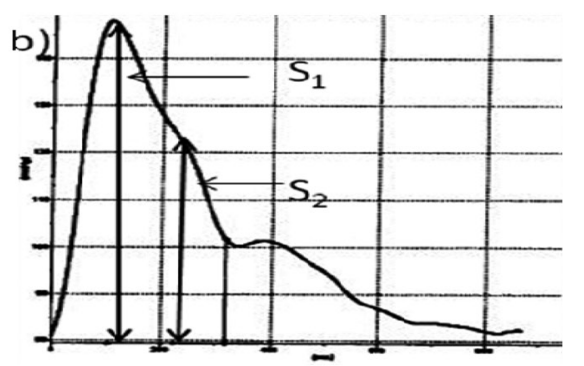

Fig. 3. A schematic representation of radial arterial waveform at a) baseline and b) 180 min post Korean red ginseng treatment in a single individual, with pulse pressure (S1) and augmentation pressure (S2) indicated. Augmentation index (dimensionless) was reported as the ratio of the pulse pressure at the second systolic peak (S2) to that at the first systolic peak (S1). 

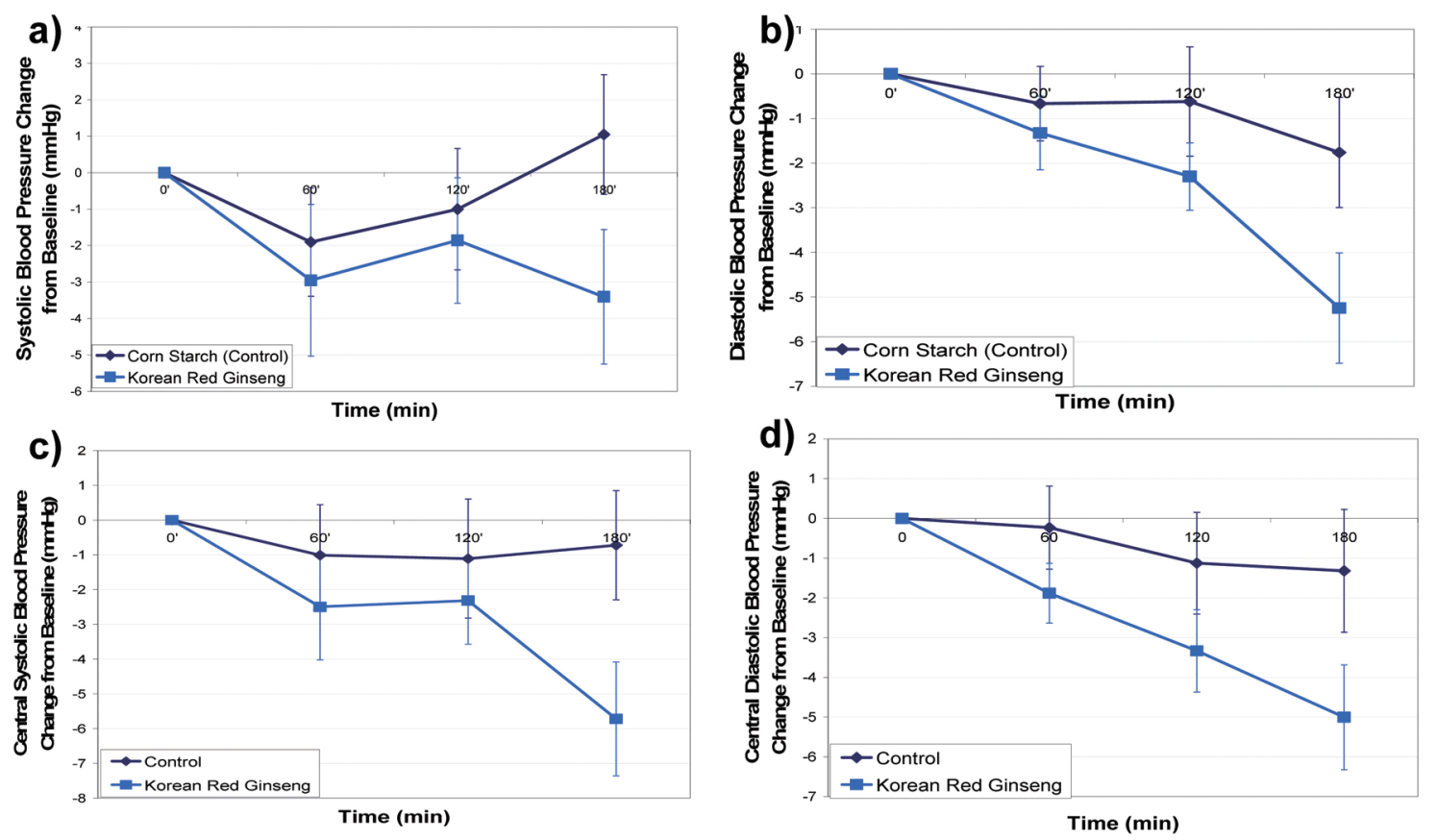

Fig. 4. The acute effect of $\mathrm{Rg}_{3}$-enriched Korean red ginseng extract on changes in vascular parameters from baseline compared to control $3 \mathrm{hr}$ post-treatment in healthy individuals. Data from a double blind randomized controlled trial: (a) systolic blood pressure change, (b) diastolic blood pressure change, (c) central systolic blood pressure change, and (d) central diastolic blood pressure change. Data is presented as mean \pm SEM.

with selected American ginseng extract in patients with diabetes and concomitant hypertension is underway.

\section{COMBINATION THERAPY: LONG TERM CLINI- CALTRIAL}

In light of the evidence of the KRG and American ginseng clinical evaluation programs for diabetes and vascular function, our ensuing intention was to consider a combination therapy with aim of advancing ginseng evaluation using a multi-targeted approach. Combination therapy has proven to be a popular treatment strategy for tighter diabetes control. Although the exact mechanism of action is unknown, based on preliminary evidence and our American and KRG screening programs, both species of ginseng act through a different, but complementary mechanism of action; American ginseng may act by increasing insulin secretion, whereas, KRG may act by improving insulin sensitivity. In addition, KRG has demonstrated clinical vasoactive potential. Therefore a long term trial was designed to investigate the therapeutic potential of American ginseng and KRG in combination to address both glycemic control via complementary action as well vascular activity potential.

The study followed a randomized, double-blind, placebo-controlled, two-centre, two-arm parallel design in individuals with type 2 diabetes. Effects on glycemic control are assessed by examining biochemical markers and the glycemic response to an oral glucose tolerance test. Additional metabolic parameters and blood pressure parameters are also evaluated as markers of CVD risk. The combined use of American ginseng and KRG for $12 \mathrm{wk}$ demonstrated a significant improvement in long term marker of glycemic control, HbA1c, by $0.4 \%$ as well as a significant improvement in systolic blood pressure by $5 \mathrm{mmHg}$ compared to placebo. The combination treatment also demonstrated to be safe, but did not significantly affect blood lipids. These findings encourage further investigation of the mechanism and roles of Korean and American ginseng combined and continuation of systematic evaluation of their effective components.

\section{CONCLUSION}

While our clinical evidence, along with traditional indication for generic ginseng is largely aligned in supporting a diabetes and possibly vascular indication, the safety and efficacy of specific ginseng sources in improving glycemic and vascular outcomes in humans remains questionable. We applied the robust systematic, "acute-to-chronic", stepwise clinical screening model to our KRG via two efficacy arms of the program. We were successful in identifying KRG sources with reproducible and sustainable glycemic benefit in diabetes as assessed 
by markers of glucose and insulin regulation. We were also successful in identifying the fractions of ginseng that have contributed to the vasoactive potential as assessed by markers of endothelial function, BP and arterial stiffness. Based on our and other laboratories, preliminary clinical studies indicate that a mechanism of action for KRG to improve diabetes control is through increase in insulin sensitivity, and to improve vascular function is through vaso-motor dilatory modification based on reflected arterial waveforms. Although our clinical screening model may provide a basis for component based "clinical standardization" of KRG, there remains a clear need to develop a better basis for standardization that ties composition directly to efficacy. More clinical work is needed to expand upon the connections that have been drawn between aspects of the ginsenoside profile, for example $\mathrm{Rg}_{3}$, of KRG and efficacy. There is also a requirement for more "phase II" trials of effectiveness are required to support clinical indications for KRG and its fractions in diabetes and vascular disease. Progress in these areas may bring us closer to establishing standardization parameters of ginseng leading to more consistent predictions of KRG efficacy.

\section{ACKNOWLEDGEMENTS}

This work was supported by grants from the Canadian Diabetes Association, Canadian Institutes of Health Research, Heart and Stroke Foundation of Canada, and Korean Ministry of Agriculture and Forestry, and BTGin Co., Chunkeon-gun, Republic of Korea. JLS was funded by a Province of Ontario Postdoctoral Fellowship, the Edie Steinberg Scholarship Fund, and the Edward Christie Stevens Fellowship in Medicine. VV has also received research funding from the Ontario Ginseng Growers Association, Simcoe, ON, Canada; and BTGin Co.

\section{REFERENCES}

1. King H, Aubert RE, Herman WH. Global burden of diabetes, 1995-2025: prevalence, numerical estimates, and projections. Diabetes Care 1998;21:1414-1431.

2. Action to Control Cardiovascular Risk in Diabetes Study Group, Gerstein HC, Miller ME, Byington RP, Goff DC Jr, Bigger JT, Buse JB, Cushman WC, Genuth S, IsmailBeigi $\mathrm{F}$, et al. Effects of intensive glucose lowering in type 2 diabetes. N Engl J Med 2008;358:2545-2559.

3. ADVANCE Collaborative Group, Patel A, MacMahon S, Chalmers J, Neal B, Billot L, Woodward M, Marre M, Cooper M, Glasziou P, et al. Intensive blood glucose control and vascular outcomes in patients with type 2 diabetes. N Engl J Med 2008;358:2560-2572.

4. Turner RC, Cull CA, Frighi V, Holman RR. Glycemic control with diet, sulfonylurea, metformin, or insulin in patients with type 2 diabetes mellitus: progressive requirement for multiple therapies (UKPDS 49). UK Prospective Diabetes Study (UKPDS) Group. JAMA 1999;281:2005-2012.

5. Nissen SE, Wolski K. Effect of rosiglitazone on the risk of myocardial infarction and death from cardiovascular causes. N Engl J Med 2007;356:2457-2471.

6. Duckworth W, Abraira C, Moritz T, Reda D, Emanuele N, Reaven PD, Zieve FJ, Marks J, Davis SN, Hayward R, et al. Glucose control and vascular complications in veterans with type 2 diabetes. N Engl J Med 2009;360:129-139.

7. Sotaniemi EA, Haapakoski E, Rautio A. Ginseng therapy in non-insulin-dependent diabetic patients. Diabetes Care 1995;18:1373-1375.

8. Sievenpiper JL, Arnason JT, Leiter LA, Vuksan V. Variable effects of American ginseng: a batch of American ginseng (Panax quinquefolius L.) with a depressed ginsenoside profile does not affect postprandial glycemia. Eur J Clin Nutr 2003;57:243-248.

9. Sievenpiper JL, Arnason JT, Leiter LA, Vuksan V. Decreasing, null and increasing effects of eight popular types of ginseng on acute postprandial glycemic indices in healthy humans: the role of ginsenosides. J Am Coll Nutr 2004; 23:248-258

10. Sievenpiper JL, Arnason JT, Leiter LA, Vuksan V. Null and opposing effects of Asian ginseng (Panax ginseng C.A. Meyer) on acute glycemia: results of two acute dose escalation studies. J Am Coll Nutr 2003;22:524-532.

11. Sievenpiper JL, Arnason JT, Vidgen E, Leiter LA, Vuksan V. A systematic quantitative analysis of the literature of the high variability in ginseng (Panax spp.): should ginseng be trusted in diabetes? Diabetes Care 2004;27:839840.

12. Pasupuleti VK, Anderson JW. Ginseng in type 2 diabetes mellitus: A review of the evidence in humans. Nutraceuticals, glycemic health and type 2 diabetes. 1st ed. Ames: Wiley-Blackwell/IFT Press, 2008.

13. Vuksan V, Sievenpiper JL. Herbal remedies in the management of diabetes: lessons learned from the study of ginseng. Nutr Metab Cardiovasc Dis 2005;15:149-160.

14. Vuksan V, Sievenpiper JL, Koo VY, Francis T, BeljanZdravkovic U, Xu Z, Vidgen E. American ginseng (Panax quinquefolius L) reduces postprandial glycemia in nondiabetic subjects and subjects with type 2 diabetes mellitus. Arch Intern Med 2000;160:1009-1013.

15. Vuksan V, Sievenpiper JL, Wong J, Xu Z, Beljan-Zdravkovic U, Arnason JT, Assinewe V, Stavro MP, Jenkins AL, Leiter 
LA, al. American ginseng (Panax quinquefolius L.) attenuates postprandial glycemia in a time-dependent but not dose-dependent manner in healthy individuals. Am J Clin Nutr 2001;73:753-758.

16. Vuksan V, Stavro MP, Sievenpiper JL, Beljan-Zdravkovic U, Leiter LA, Josse RG, Xu Z. Similar postprandial glycemic reductions with escalation of dose and administration time of American ginseng in type 2 diabetes. Diabetes Care 2000;23:1221-1226.

17. Vuksan V, Stavro MP, Sievenpiper JL, Koo VY, Wong E, Beljan-Zdravkovic U, Francis T, Jenkins AL, Leiter LA, Josse RG, et al. American ginseng improves glycemia in individuals with normal glucose tolerance: effect of dose and time escalation. J Am Coll Nutr 2000;19:738-744.

18. Vuksan V, Sievenpiper JL, Xu Z, Wong EY, Jenkins AL, Beljan-Zdravkovic U, Leiter LA, Josse RG, Stavro MP. Konjac-Mannan and American ginsing: emerging alternative therapies for type 2 diabetes mellitus. J Am Coll Nutr 2001;20(5 Suppl):370S-380S.

19. Stavro PM, Woo M, Heim TF, Leiter LA, Vuksan V. North American ginseng exerts a neutral effect on blood pressure in individuals with hypertension. Hypertension 2005; 46:406-411.

20. Stavro PM, Woo M, Leiter LA, Heim TF, Sievenpiper JL, Vuksan V. Long-term intake of North American ginseng has no effect on 24-hour blood pressure and renal function. Hypertension 2006;47:791-796.

21. Tetsutani T, Yamamura M, Yamaguchi T, Onoyama O, Kono M. Can red ginseng control blood glucose in diabetic patients. Ginseng Rev 2000;28:44-47.

22. Sievenpiper JL, Sung MK, Di Buono M, Seung-Lee K, Nam KY, Arnason JT, Leiter LA, Vuksan V. Korean red ginseng rootlets decrease acute postprandial glycemia: results from sequential preparation- and dose-finding studies. J Am Coll Nutr 2006;25:100-107.

23. Vuksan V, Sung MK, Sievenpiper JL, Stavro PM, Jenkins AL, Di Buono M, Lee KS, Leiter LA, Nam KY, Arnason JT, et al. Korean red ginseng (Panax ginseng) improves glucose and insulin regulation in well-controlled, type 2 diabetes: results of a randomized, double-blind, placebocontrolled study of efficacy and safety. Nutr Metab Cardiovasc Dis 2008;18:46-56.

24. Zhou W, Chai H, Lin PH, Lumsden AB, Yao Q, Chen CJ. Molecular mechanisms and clinical applications of ginseng root for cardiovascular disease. Med Sci Monit 2004;10:RA187-RA192.

25. Yun TK. Brief introduction of Panax ginseng C.A. Meyer. J Korean Med Sci 2001;16 Suppl:S3-S5.

26. Kang SY, Kim SH, Schini VB, Kim ND. Dietary ginsenosides improve endothelium-dependent relaxation in the thoracic aorta of hypercholesterolemic rabbit. Gen Pharmacol 1995;26:483-487.

27. Kang SY, Schini-Kerth VB, Kim ND. Ginsenosides of the protopanaxatriol group cause endothelium-dependent relaxation in the rat aorta. Life Sci 1995;56:1577-1586.

28. Kim ND, Kang SY, Kim MJ, Park JH, Schini-Kerth VB. The ginsenoside $\mathrm{Rg}_{3}$ evokes endothelium-independent relaxation in rat aortic rings: role of $\mathrm{K}+$ channels. Eur $\mathrm{J}$ Pharmacol 1999;367:51-57.

29. Kim ND, Kang SY, Park JH, Schini-Kerth VB. Ginsenoside $\mathrm{Rg}_{3}$ mediates endothelium-dependent relaxation in response to ginsenosides in rat aorta: role of $\mathrm{K}+$ channels. Eur J Pharmacol 1999;367:41-49.

30. Kim ND, Kang SY, Schini VB. Ginsenosides evoke endothelium-dependent vascular relaxation in rat aorta. Gen Pharmacol 1994;25:1071-1077.

31. Kim ND, Kim EM, Kang KW, Cho MK, Choi SY, Kim SG. Ginsenoside $\mathrm{Rg}_{3}$ inhibits phenylephrine-induced vascular contraction through induction of nitric oxide synthase. Br J Pharmacol 2003;140:661-670.

32. Sung J, Han KH, Zo JH, Park HJ, Kim CH, Oh BH. Effects of red ginseng upon vascular endothelial function in patients with essential hypertension. Am J Chin Med 2000;28:205-216.

33. Park KM, Kim YS, Jeong TC, Joe CO, Shin HJ, Lee YH, Nam KY, Park JD. Nitric oxide is involved in the immunomodulating activities of acidic polysaccharide from Panax ginseng. Planta Med 2001;67:122-126.

34. Shin JY, Song JY, Yun YS, Yang HO, Rhee DK, Pyo S. Immunostimulating effects of acidic polysaccharides extract of Panax ginseng on macrophage function. Immunopharmacol Immunotoxicol 2002;24:469-482.

35. Han K, Shin IC, Choi KJ, Yun YP, Hong JT, Oh KW. Korea red ginseng water extract increases nitric oxide concentrations in exhaled breath. Nitric Oxide 2005;12:159162.

36. Han KH, Choe SC, Kim HS, Sohn DW, Nam KY, Oh BH, Lee MM, Park YB, Choi YS, Seo JD, et al. Effect of red ginseng on blood pressure in patients with essential hypertension and white coat hypertension. Am J Chin Med 1998;26:199-209.

37. Caron MF, Hotsko AL, Robertson S, Mandybur L, Kluger J, White CM. Electrocardiographic and hemodynamic effects of Panax ginseng. Ann Pharmacother 2002;36:758763.

38. Nichols WW, Edwards DG. Arterial elastance and wave reflection augmentation of systolic blood pressure: deleterious effects and implications for therapy. J Cardiovasc Pharmacol Ther 2001;6:5-21.

39. Nichols WW, Singh BM. Augmentation index as a meas- 
ure of peripheral vascular disease state. Curr Opin Cardiol 2002;17:543-551.

40. Laurent S, Boutouyrie P, Asmar R, Gautier I, Laloux B, Guize L, Ducimetiere P, Benetos A. Aortic stiffness is an independent predictor of all-cause and cardiovascular mortality in hypertensive patients. Hypertension 2001;37:12361241.

41. Felmeden DC, Spencer CG, Blann AD, Beevers DG, Lip GY. Low-density lipoprotein subfractions and cardiovascular risk in hypertension: relationship to endothelial dysfunction and effects of treatment. Hypertension 2003;41:528533.

42. Stavro PM, Woo M, Vuksan V. Korean red ginseng lowers blood pressure in individuals with hypertension. Am J Hypertens 2004; 17:33A.
43. Stavro PM, Hana AK, Vuksan V. The effect of Korean red ginseng extracts with escalating levels of ginsenoside $\mathrm{Rg}_{3}$ on blood pressure in individuals with high normal blood pressure or hypertension. Am J Hypertens 2002; 15:34A.

44. Jovanovski E, DeSouza L, Ezatagha A, Lee AS, Jenkins AL, Dias A, Vuksan V. The Influence of Korean red ginseng (Panax ginseng C. A. Mayer) root and its derived fractions, ginsenosides and polysaccharides on endothelial function: an acute double blind, crossover randomized controlled trial in healthy individuals. In: Natural Health Product Research Society of Canada. Proceeding of the 5 th annual natural health products research conference and trade show: science across borders, global natural health products research; 2008 Mar 26-29; Toronto, ON, Canada. March $29^{\text {th }}$ Oral Presentation. 